Manuscript ID

ZUMJ-2105-2238 (R1)

DOI

$10.21608 /$ zumj.2021.77150.2238

ORIGINAL ARTICLE

\title{
Role of GRK5/ $\beta$-arrestin2 pathway in mediating the cardioprotective effects of carvedilol in 5/6 nephrectomized rats
}

\author{
Rasha M.S.M. Mohamed ${ }^{\text {a*}}$, Shimaa Elshazly ${ }^{\text {b }}$, Amal E. Salem ${ }^{\text {a }}$ and Nevertyty Mahmoud ${ }^{\text {a }}$ \\ ${ }^{a}$ Clinical Pharmacology Department, Faculty of Medicine, Zagazig University, Zagazig, 44519 Egypt \\ b Pharmacology and Toxicology Department, Faculty of Pharmacy, Zagazig University, Zagazig, \\ 44519 Egypt.
}

\author{
Corresponding author: \\ Rasha Mohamed Sabry Mohamed \\ Mohamed \\ Clinical Pharmacology \\ Department, Faculty of Medicine, \\ Zagazig, University. \\ E-mail: \\ rmsabry@medicine.zu.edu.eg \\ ORCID Id: 0000-0002-4173-4399
}

$\begin{array}{ll}\text { Submit Date } & \text { 2021-05-22 18:23:27 } \\ \text { Revise Date } & 2021-06-1919: 07: 41 \\ \text { Accept Date } & 2021-08-0214: 55: 35\end{array}$

\section{INTRODUCTION}

C ardio-renal syndrome (CRS) describes the mutual effects of both cardiac and renal injury on each other. Patients suffering from heart failure are usually suffering from renal failure and vice versa [1]. The cardiorenal syndrome is classified into five types. In this study, we induced type 4 CRS, also known as chronic reno-cardiac syndrome, by inducing $5 / 6$ nephrectomy in rats. This type is usually associated with heart failure, cardiac hypertrophy, and cardiac remodeling [2].

The exact mechanisms by which the heart and kidney affect each other are not fully understood. Many reports suggested a possible role for central venous congestion [3], neurohormonal involvement [4], oxidative stress [5],

\section{ABSTRACT}

Background: Cardiorenal-syndrome is associated with high morbidity amlexanox groups. Rats were subjected to $5 / 6$ nephrectomy. Just after surgery, drugs were given for 10 weeks. At the end of study, kidney functions, blood pressure, and markers of cardiomyocyte apoptosis and heart failure were measured. Also, cardiac $\beta 1$-adrenergic receptor cardioprotective-effects. Conclusion: Carvedilol-induced cardiac protection in 5/6 nephrectomized rats is highly correlated with upregulation of cardiac G-protein coupled receptor kinase $5 / \beta$-arrestin2 pathway.

Keywords: Carvedilol - $\beta$-arrestin2 - cardiorenal syndrome - amlexanox - G-protein coupled receptor kinase 5(GRK5) and renal sympathetic system activity [6] as potential contributors. Research groups working on the renal sympathetic activity, as a possible cause of this syndrome, use $\beta$ blockers, angiotensin receptor blockers (ARBs), and their combination as potential treatments for this syndrome [1].

Carvedilol is a non-selective $\beta$ blocker with $\alpha_{1}$-blocking effects [7] that can protect the heart by blocking the detrimental effects of circulating catecholamines [8]. Previous studies have shown that carvedilol is more effective in the management of heart failure than other $\beta$-blockers. Later, studies revealed that $\beta$-arrestin dependent signals are responsible for these beneficial effects of carvedilol. Therefore, carvedilol is described 
as a $\beta$-arrestin biased agonist with selective activation of G-protein coupled receptor (GPCR)- $\beta$-arrestin signaling, while blocks GPCR-G-protein pathway [9].

$\beta$-Arrestin protein is first named for its ability to arrest (desensitize) $\beta$-adrenergic receptors by uncoupling G-proteins. It was confirmed that $\beta$-arrestins can stimulate its own downstream signaling pathway independent of G-protein pathways [10]. Moreover, studies revealed that $\beta$-arrestin dependent signals in the heart can enhance cardiac contractility and cardiomyocytes survival in addition to inhibition of apoptotic signals activated by G-protein pathways [11]. Activation of $\beta$-arrestin signaling requires phosphorylation of the GPCR with GPCR kinase 5 or 6 (GRK5 or GRK6) [12].

Phosphatidylinositol 4, 5 bisphosphate (PIP2) is a down-stream signal for both G-proteins and $\beta$-arrestins. Notably, G $\alpha$ q and G $\beta \gamma$-proteins can breakdown PIP2 into diacylglycerol (DAG) and inositol triphosphate (IP3) through activation of phospholipase-C (PLC) [13]. On the other hand, $\beta$-arrestins can promote PIP 2 production [14]. In addition, phosphorylation of PIP2 into PIP3 activates protein kinase $\mathrm{B}$ (PKB or AKT) [15], which acts as a survival signal by deactivation of glycogen synthase kinase-3 $\beta$ (GSK3 $\beta)$ [16].

This work investigated the correlation between the GRK5/ $\beta$-arrestin2 pathway and the cardioprotective effects of carvedilol in $5 / 6$ nephrectomized rats.

\section{MATERIALS AND METHODS}

\section{Animals and ethics statement}

Adult male Wistar rats with an average weight of about 240 to 260 grams were bought from the Faculty of Veterinary Medicine, Zagazig University, Egypt. Rats were distributed three/cage. They were kept for one week as an acclimatization period. Rats had free access to diet and water. Average temperature $\left(23 \pm 2^{\circ} \mathrm{C}\right)$, light/dark cycles $(12 / 12$ h), and humidity $(60 \pm 10 \%)$ were kept constant. Animal handling approaches were validated by the Ethical Committee for Animal Handling at the Faculty of Pharmacy, Zagazig University, Egypt, possessing approval no. P4-8-2017. All animal experiments comply with the ARRIVE guidelines and are carried out in accordance with the U.K. Animals guidelines.

\section{Drugs and experimental design}

There were five groups: sham group $(\mathrm{n}=9), 5 / 6$ nephrectomy group $(\mathrm{n}=15)$, carvedilol group $(\mathrm{n}=10)$, amlexanox group $(\mathrm{n}=10)$ and carvedilol + amlexanox group $(n=10)$. In the sham group, kidneys underwent evacuation, decapsulation then returned into the abdomen. This procedure was performed bilaterally, two-week apart. In the 5/6 nephrectomy group rats were subjected to surgical operation and removal of $5 / 6$ of the renal mass. In the carvedilol group, 5/6 nephrectomized rats received carvedilol (20mg/kg/day, i.p., Roche, Mannheim, Germany, [17]). In the amlexanox group, 5/6 nephrectomized rats received amlexanox, a Gprotein coupled receptor kinase 5 inhibitor (GRK5i) (25mg/kg/day, i.p., Sigma, St. Louis, MO, [18]). In the carvedilol + amlexanox group, 5/6 nephrectomized rats received amlexanox then carvedilol after 30 minutes in the same doses and route of administration previously mentioned. All drugs were dissolved in 5\% dimethyl sulfoxide (DMSO, diluted with saline) and given for 10 weeks just after surgery. Also, sham and 5/6 nephrectomy groups received 5\% DMSO in the same volume and sequence of treated groups (shown in Fig. 1)

\section{Induction of renal failure by $5 / 6$ nephrectomy of total renal mass}

First, a lateral dorsal incision was done to expose and decapsulate the left kidney. After that, clamping renal vessels and dissecting both poles ( $2 / 3$ of the functional left kidney mass). Then, instant glue was used to treat the cut surface and stop bleeding. At last, removal of vessel clamp and returning the kidney stump into the abdomen was done. Two weeks later, a total nephrectomy of the right kidney was done through a lateral dorsal incision after ligating the right renal vessels. All operations, in sham and 5/6 nephrectomy groups were performed under total anesthesia using sodium pentobarbital (100 mg/kg, i.p.). All procedures were performed under strict aseptic conditions. The adrenal glands were cautiously protected from damage during the operations. Finally, the lateral dorsal incision 
was sutured in two layers and fixed by instant glue. Following the operation, each rat received an antibiotic, Benzyl Penicillin (Penicillin G Sodium) (AQUA - PEN 1000.000 I.U, CID, Egypt) and kept in a separate cage freely accessing food and water. Renal failure was significant after 10 days of surgery while heart failure and cardiac remodeling was significant after 10 weeks of surgery [19].

\section{Measurement of blood pressure}

Blood pressure was estimated using a tail-cuff blood pressure measuring system. Blood pressure was monitored from 10 AM to 12 noon, by non-invasive tail-cuff method (Harvard Apparatus Ltd, Edenbridge, Kent, England). Rats were trained with the instrument for one week before initiation of the experiment and were conscious during the measurement. Rats were placed in a heated restrainer at $37 \pm 1{ }^{\circ} \mathrm{C}$ for 10 minutes. At least 3 blood pressures were measured for each rat and the average of them was reported. Blood pressure was measured before surgery, 10 days after surgery and at the end of the experiment.

\section{Collection of blood and serum separation}

At the end of the experiment, all animals received urethane $(1.3 \mathrm{~g} / \mathrm{kg}$, i.p., Sigma-Aldrich, USA) to achieve anesthesia. Collecting blood samples was done from the orbital sinus of fasting rats. Immediately, blood samples underwent centrifugation to separate serum then stored at $-20^{\circ} \mathrm{C}$. Blood samples were also collected before surgery and 10 days after surgery as well.

\section{Collection of heart samples for laboratory analysis:}

Finally, heart samples were collected after the decapitation of rats. Heart samples were kept immediately in liquid nitrogen at $80^{\circ} \mathrm{C}$. The weights of the hearts was recorded and normalized by tibial length.

\section{Colorimetric assays}

Measuring serum creatinine and serum urea was done using quantitative colorimetric assay kits, commercially available, purchased from Bio-diagnostic Co. (Egypt). Caspase-3 activity assay kit (Sigma Aldrich, USA) was used to measure cardiac Caspase-3 activity. All procedures followed the instructions of the manufacturer.

\section{Enzyme-linked immunosorbent assays (ELISA)}

Serum levels of brain natriuretic peptide (BNP, Peninsula Laboratories, Bachem Group, USA) and cardiac levels of $\beta 1$ adrenergic receptor ( $\beta 1 \mathrm{AR}$, Biomatik, Ontario, Canada), $\beta$-arrestin2 (life span biosciences, Inc.), phosphatidylinositol 4,5 bisphosphate (PIP2, Echelon Biosciences, Inc., Salt Lake City, UT), diacylglycerol (DAG, Wuhan EIAab Science Co., Ltd, China) and phosphorylated AKT S473 (Kit-3997, DRG International, Inc. USA) were estimated using rat ELISA kits. All procedures were done according to the instructions of the manufacturer.

\section{Statistics}

Data are presented as mean $\pm \mathrm{SE}$. Groups were analyzed using two-way ANOVA and post-hoc Bonferroni, or one-way ANOVA followed by post-hoc Tukey test by GraphPad Prism version 5 (GraphPad Software, Inc., CA, USA). P-values $<0.05$ are considered significant.

\section{RESULTS \\ Effect on kidney functions and blood pressure}

In Figure 2, 5/6 nephrectomized rats experienced significant increases in serum urea $(110.1 \pm 7.6$ vs $24.8 \pm 3.1 \mathrm{mg} / \mathrm{dl}$ after 10 days and $125.9 \pm 7.9$ vs $23.8 \pm 2.8 \mathrm{mg} / \mathrm{dl}$ after 10 weeks of surgery) and serum creatinine $(0.87 \pm 0.04$ vs $0.52 \pm 0.03 \mathrm{mg} / \mathrm{dl}$ after 10 days and $0.99 \pm 0.03$ vs $0.58 \pm 0.01 \mathrm{mg} / \mathrm{dl}$ after 10 weeks of surgery) in comparison to the sham group (Fig. 2. A, B). In contrast, serum urea level significantly decreased in the carvedilol treated group $(-14.5 \%$ and $-56.8 \%$ after 10 days and 10 weeks respectively) compared to 5/6 nephrectomized rats. Moreover, serum creatinine level significantly decreased in the carvedilol treated group $(-11 \%$ and $-30 \%$ after 10 days and 10 weeks respectively) compared to 5/6 nephrectomized rats. Moreover, preinjection of amlexanox (GRK5i) to carvedilol significantly increased both serum creatinine $(14 \%)$ and serum urea (30\%) levels after 10 weeks of surgery in comparison to the carvedilol group. (Fig. 2. A, B)

Regarding effects on blood pressure, 5/6 Nephrectomized rats showed significant 
increases in diastolic blood pressure $(124 \pm 9.6$ vs $91 \pm 5.9$ after 10 days and $129 \pm 5.6$ vs $84 \pm 3.8$ after 10 weeks of surgery, respectively) and systolic blood pressure $(179.2 \pm 11.4$ vs $118.3 \pm 7.8$ after 10 days and $200.8 \pm 15.5$ vs $124 \pm 7.7$ after 10 weeks of surgery, respectively) compared to the sham group (Fig. 2. C, D). In contrast, carvedilol significantly decreased diastolic blood pressure (-15\% and $-21 \%)$ after 10 days and 10 weeks of surgery compared to $5 / 6$ nephrectomized rats. While carvedilol significantly reduced systolic blood pressure after 10 days and 10 weeks of surgery $(-22.3 \%$ and $-30 \%$ ) compared to $5 / 6$ nephrectomized rats. Moreover, pre-injection of amlexanox (GRK5i) to carvedilol increased both diastolic (23\%, $\mathrm{P}=0.055$, after 10 weeks) and systolic blood pressures $(23.5 \% \quad(\mathrm{P}<0.05)$ after 10 weeks) compared to the carvedilol group. (Fig. 2. C, D)

Effect on cardiac hypertrophy and serum BNP level (as a marker of heart failure):

In 5/6 nephrectomized rats, there was a significant increase in normalized heart weights to tibial length $(428 \pm 9.6$ vs $283.8 \pm 6$ $\mathrm{mg} / \mathrm{cm})$ and serum BNP $(2.04 \pm 0.09$ vs $0.26 \pm 0.02 \mathrm{ng} / \mathrm{ml}$ ) levels compared to the sham group (Fig.3. A, B respectively). In contrast, carvedilol significantly decreased heart weights (-23\%) and serum BNP (-76\%) levels compared to the $5 / 6$ nephrectomy group (Fig.3. A, B respectively). Moreover, preinjection of amlexanox (GRK5i) to carvedilol significantly increased both heart weights $(22 \%)$ and serum BNP (275\%) levels compared to the carvedilol group (Fig.3. A, B respectively).

Effect on cardiac $\beta 1$-adrenergic receptors ( $\beta 1 A R s)$ and down-stream signals

5/6 Nephrectomized rats showed significant reductions in cardiac levels of $\beta 1$ ARs $(13.8 \pm 1.03$ vs $38.6 \pm 2.6$ pg/mg, Fig. 4. A), $\beta$-arrestin2 $(81.8 \pm 5.5$ vs $166.8 \pm 9.7 \mathrm{pg} / \mathrm{mg}$,
Fig. 4. B) and PIP2 (16.2 \pm 1.5 vs $47 \pm 3.2$ $\mathrm{nmol} / \mathrm{mg}$, Fig. 4. C) levels in comparison to sham group. Moreover, 5/6 nephrectomized rats showed significant increases in cardiac DAG (5.3 \pm 0.33 vs $1.64 \pm 0.12$, Fig. 4. D) levels in comparison to the sham group. In contrast, carvedilol significantly increased cardiac levels of $\beta 1$ ARs $(361 \%)$ (Fig. 4. A), $\beta$ arrestin2 (111\%) (Fig. 4. B) and PIP2 (156\%) (Fig. 4. C) levels compared to $5 / 6$ nephrectomy group. Also, carvedilol significantly decreased cardiac levels of DAG $(-67 \%)$ (Fig. 4. D) compared to $5 / 6$ nephrectomy group. Moreover, pre-injection of amlexanox (GRK5i) to carvedilol significantly reduced cardiac tissue levels of $\beta 1$ ARs (-21.6\%), $\beta$-arrestin2 (-52\%), and PIP2 $(-46 \%)$ compared to the carvedilol group (Fig. 4. A, B and C, respectively). Besides, preinjection of amlexanox (GRK5i) to carvedilol significantly increased cardiac DAG level (145\%) compared to the carvedilol group (Fig. 4. D).

\section{Effect on cardiac apoptotic pathways}

5/6 Nephrectomized rats showed significant decreases in cardiac pS473 AKT level, the active form of AKT, indicating reduced AKT activity $(1.8 \pm 0.15$ vs $5.1 \pm 0.3$ $\mathrm{ng} / \mathrm{mg}$ ) (Fig. 5. A) and significant increases in cardiac caspase- 3 activity $(453.7 \pm 32$ vs $79.7 \pm 9.7 \mathrm{U} / \mathrm{g}$, Fig. 5. B) compared to the sham group. In contrast, carvedilol significantly elevated cardiac pS473AKT level, indicating increased AKT activity (88\%) (Fig. 5. A) and significantly decreased cardiac caspase-3 activity (-65\%) (Fig. 5. B) compared to the $5 / 6$ nephrectomy group. Moreover, pre-injection of amlexanox (GRK5i) to carvedilol significantly decreased cardiac pS473AKT level, indicating reduced AKT activity (-35\%) concomitant with a significant increase in cardiac caspase 3 activity compared to the carvedilol group (144\%) (Fig. 5. A, B). 


\section{Figure 1}

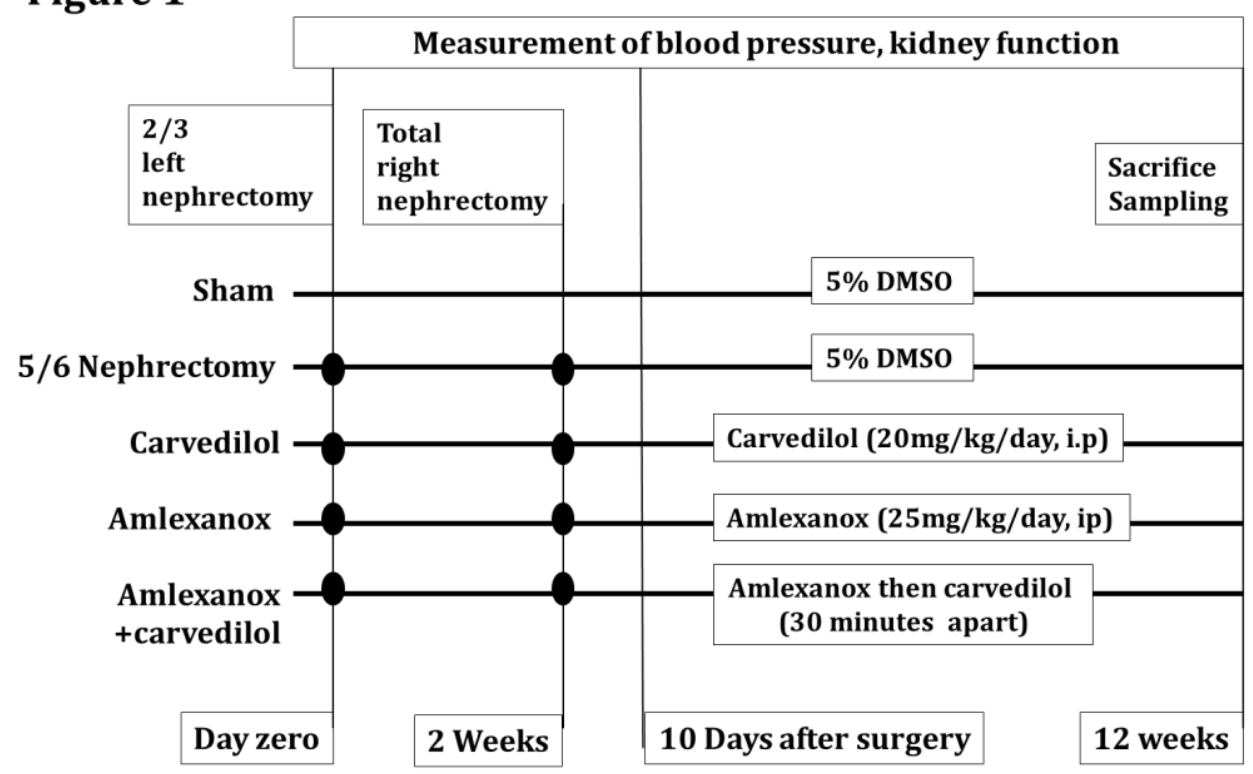

Fig. 1. Experimental design.

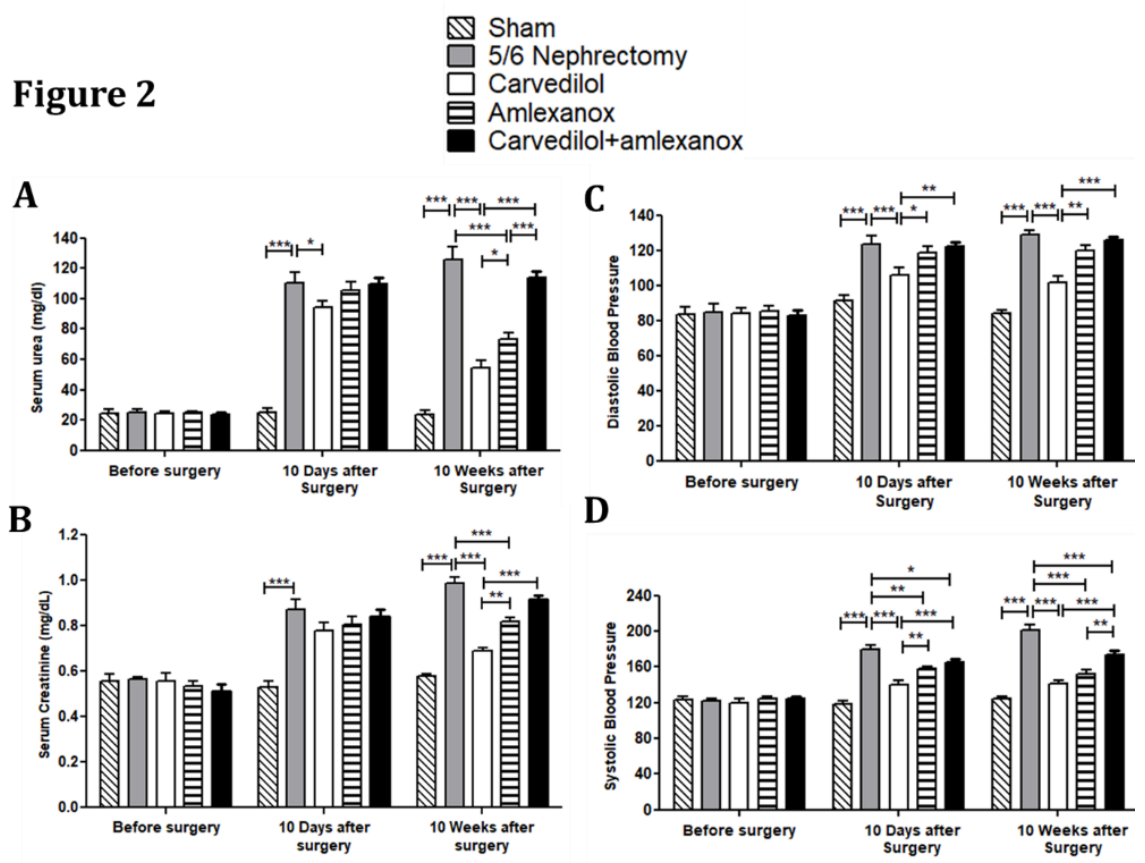

Fig. 2. Effect of carvedilol, amlexanox, and their combination on kidney functions and blood pressure after 10 days and 10 weeks in 5/6 nephrectomized rats. Graphical presentation of serum urea (A), serum creatinine (B), Tail cuff reads of diastolic (C) and systolic (D) blood pressures. Carvedilol (20 mg/kg/day) and amlexanox $(25 \mathrm{mg} / \mathrm{kg} /$ day $)$ were injected i.p. for 10 weeks, starting just after $5 / 6$ nephrectomy. In the carvedilol + amlexanox group, carvedilol was injected 30 minutes after injection of amlexanox. Groups were analyzed using two-way ANOVA and post-hoc Bonferroni's test. All values were shown as mean \pm SE. $(\mathrm{n}=5)$. $* \mathrm{P}<0.05, * * \mathrm{P}<0.01$, and $* * * \mathrm{P}<0.001$. 


\section{Figure 3}
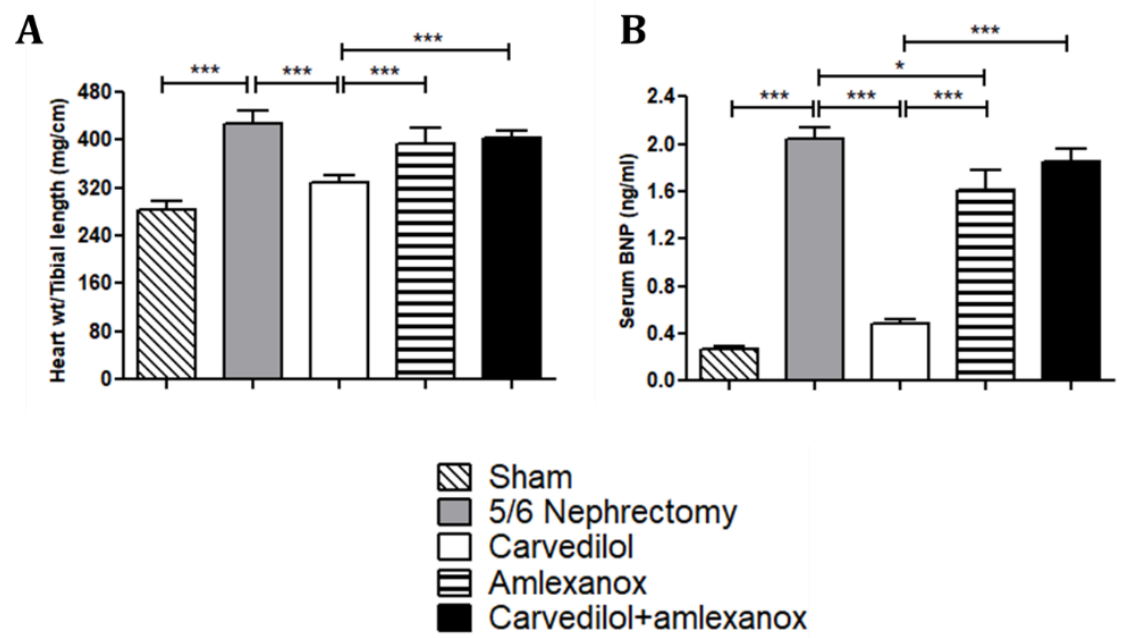

Fig. 3. Effects of carvedilol, amlexanox, and their combination on heart weights, and serum brain natriuretic peptide (BNP) after 10 weeks of treatment in 5/6 nephrectomized rats. Graphical presentation of Heart weight/tibial length (A) and serum BNP (B). Carvedilol (20 mg/kg/day) and amlexanox $(25 \mathrm{mg} / \mathrm{kg} /$ day $)$ were injected i.p. for 10 weeks, starting just after $5 / 6$ nephrectomy. In the carvedilol + amlexanox group, carvedilol was injected 30 minutes after injection of amlexanox. Groups were compared by one-way ANOVA and post-hoc Tukey's test. Values were shown as mean \pm SE. $(n=5)$. $* \mathrm{P}<0.05$, and $* * * \mathrm{P}<0.001$.

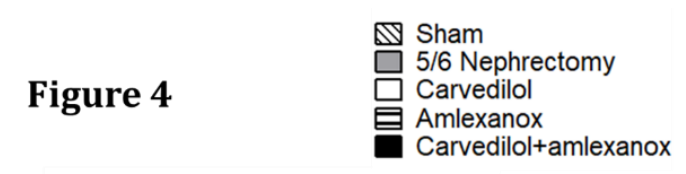

\section{A}

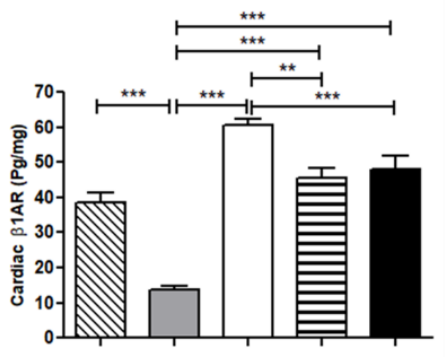

B

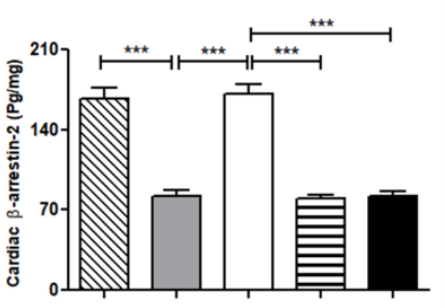

C

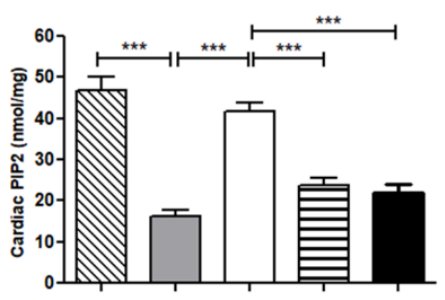

D

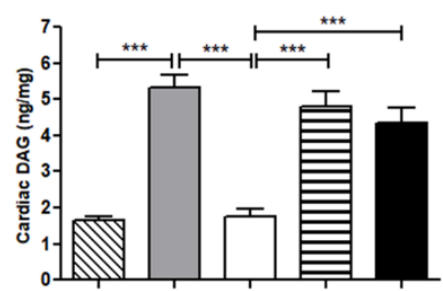

Fig. 4. Effects of carvedilol, amlexanox, and their combination on cardiac adrenergic signaling pathways after 10 weeks of treatment in $5 / 6$ nephrectomized rats. Graphical presentation of cardiac $\beta 1$ adrenergic receptors ( $\beta 1 \mathrm{ARs})(\mathrm{A}), \beta$-arrestin $2(\mathrm{~B})$, phosphatidylinositol 4,5 bisphosphate (PIP2) (C) and diacylglycerol (DAG) (D). Carvedilol (20 mg/kg/day) and amlexanox $(25 \mathrm{mg} / \mathrm{kg} /$ day $)$ were injected i.p. for 10 weeks, starting just after 5/6 nephrectomy. In the carvedilol + amlexanox group, carvedilol was injected 30 minutes after injection of amlexanox. Groups were compared by one-way ANOVA and post-hoc Tukey's test. Values were shown as mean \pm SE. $(\mathrm{n}=5)$. $* * * \mathrm{P}<0.001$. 


\section{Figure 5}
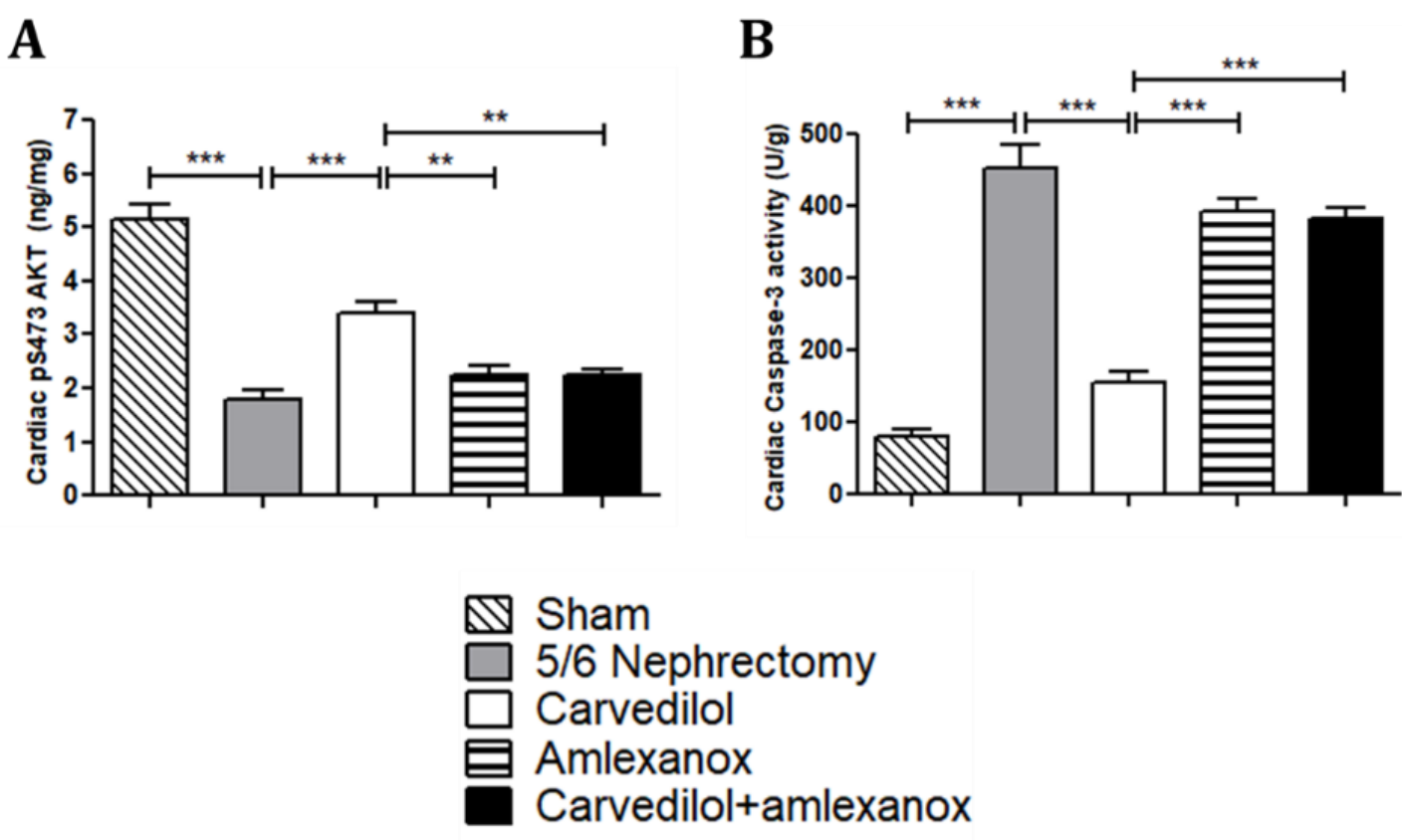

Fig. 5. Effects of carvedilol, amlexanox, and their combination on cardiac apoptotic signals after 10 weeks of treatment in 5/6 nephrectomized rats. Graphical presentation of cardiac pS473AKT (AKT) and caspase-3 activity (B). Carvedilol (20 mg/kg/day) and amlexanox ( $25 \mathrm{mg} / \mathrm{kg} /$ day) were injected i.p. for 10 weeks, starting just after 5/6 nephrectomy. In the carvedilol + amlexanox group, carvedilol was injected 30 minutes after injection of amlexanox. Groups were compared by one-way ANOVA and post-hoc Tukey's test. Values were shown as mean \pm SE. $(\mathrm{n}=5)$. ${ }^{* *} \mathrm{P}<0.01$, and $* * * \mathrm{P}<0.001$.

\section{DISCUSSION}

Chronic kidney disease (CKD) is one of the worldwide health problems with high morbidity and mortality rates. Patients suffering from CKD are usually suffering from cardiovascular complications, a condition known as the cardiorenal syndrome (CRS) [1]. Molecular mechanisms of CRS are not fully understood. Notably, $\beta$-arrestin biased agonists are promising agents in protection against heart failure [11]. This study tried to explore the correlation between the GRK5/ $\beta$ arrestin2 pathway and the cardioprotective effects of carvedilol in a rat model of CRS.

Cardiorenal syndrome type 4 was induced by performing $5 / 6$ nephrectomy in rats, a model that induces hypertension after 10 days and cardiac remodeling after 10 weeks [19]. In agreement with (Švíglerová et al., 2010) [19], our results showed significant elevation in serum creatinine and urea levels compared to the sham group after 10 days and
10 weeks of 5/6 nephrectomy. Also, our results showed a significant rise in both diastolic and systolic blood pressures compared to the sham group after 10 days and 10 weeks. Moreover, heart weights significantly increased indicating cardiac hypertrophy. Furthermore, serum BNP levels, a marker of heart failure [20], significantly increased compared to sham group [21].

One of the potential causes involved in cardiorenal syndrome is over activation of renal sympathetic system [6]. Therefore, $\beta$ blockers are commonly used for the management of this syndrome.

Selective targeting of $\beta$-arrestin down-stream signals is promising in the management of different types of cardiac disorders [22]. There are two isoforms of $\beta$ arrestins, 1 and 2. $\beta$-arrestin2 isoform is thought to mediate cardioprotective effects [10]. To investigate the role of renal sympathetic activity and $\beta$-arrestin 2 in 
cardiorenal syndrome, we measured changes in cardiac expression levels of both $\beta 1$ ARs and $\beta$-arrestin 2 in $5 / 6$ nephrectomized rats.

Our results showed significant reductions in both $\beta 1 \mathrm{ARs}$ and $\beta$-arrestin2 levels in the hearts of $5 / 6$ nephrectomized rats compared to the sham group. In agreement with our findings, Meier and colleagues showed that $\beta$ ARs are downregulated after prolonged exposure to epinephrine [23]. Also, Ungerer and colleagues reported similar observations in failing human hearts [24]. On the other hand, previous studies that measured $\beta$-arrestin2 levels in failing hearts showed wide variations depending on the model used and the site of detection.

Ungerer and colleagues showed no significant changes in $\beta$-arrestin 2 levels in failing human hearts [25]. While Vinge and colleagues showed significant initial increases in $\beta$-arrestin2 mRNA levels followed by a decline to normal values after 42 days in failing hearts using left coronary artery ligation model [26]. On the other hand, Philip and colleagues showed significant increases in $\beta$ arrestin2 levels in cardiac fibroblasts isolated from adult failing human left ventricles [27].

In the same context, our results showed a significant reduction in $\beta$-arrestin2 down-stream signals including PIP2 and pAKT levels in the heart of 5/6 nephrectomized rats in comparison to the sham group. Moreover, substrates that are blocked by $\beta$-arrestin 2 signaling were elevated like DAG and caspase- 3 activity, confirming the potential role of $\beta$-arrestin 2 in this model.

PIP2 is a down-stream signal for both G-proteins and $\beta$-arrestins. Gaq and G $\beta \gamma$ proteins can mediate PIP2 breakdown into DAG and IP3 through activation of PLC [13]. G $\beta \gamma$-protein is associated with all types of GPCRs including Gas-PCRs like $\beta 1$ ARs [28]. Therefore, low PIP2 and high DAG cardiac levels may indicate high G-proteins activity and over-stimulation of GPCRs like $\beta 1$ ARs in the heart of $5 / 6$ nephrectomized rats. $\beta$ Arrestins can promote PIP2 production by uncoupling of G-proteins or possibly by other direct effects [14]. In agreement with our findings, Suresh and colleagues reported that activation of PLC and depletion of PIP2 contributes to heart failure by compromising T-tubule function [2].

Noteworthy, DAG is an upstream signal for protein kinase C (PKC) [29]. Activation of PKC mediates cardiac hypertrophy, remodeling, and failure [30]. Braz and colleagues showed that transgenic overexpression of $\mathrm{PKC} \alpha$ promotes ventricular dysfunction and alterations in $\mathrm{Ca}^{2+}$ homeostasis [31].

Furthermore, phosphorylation of PIP2 to PIP3 promotes activation of AKT protein with subsequent inhibition of caspase3 leading to decreased apoptosis' [16, 32] Therefore, depletion of cardiac PIP2 level as observed in 5/6 nephrectomized rats contributes to increased cardiomyocytes apoptosis. In agreement with our results, chang and colleagues showed reduced cardiac AKT and increased caspase- 3 activities in a heart failure model in rats [33].

To investigate the potential role of $\beta$ arrestin 2 in our model, we explored the effect of carvedilol as a $\beta$-arrestin biased agonist [9] on cardiac remodeling in 5/6 nephrectomized rats. Carvedilol can block sympathetic effects on $\beta 1$ ARs. However, carvedilol can mediate additional effects by selective activation of $\beta$ arrestins down-stream signals.

Our results showed that carvedilol improved kidney functions compared to the $5 / 6$ nephrectomy group. This effect may be attributed to the blocking of sympathetic activity in the kidney or indirectly to improved cardiac functions. Also, carvedilol reduced diastolic and systolic blood pressures, heart weights, and serum BNP levels compared to the 5/6 nephrectomy group. In agreement with our findings, previous studies showed that use of $\beta$-blockers can protect against heart failure by blocking detrimental effects of sympathetic over-activity [34].

In the same context, carvedilol elevated cardiac levels of $\beta 1 \mathrm{AR}$ and $\beta$ arrestin 2 compared to the $5 / 6$ nephrectomy group. In agreement with our findings, previous studies confirmed that, prolonged use of $\beta$-blockers is associated with increased $\beta$ receptors expression [35].

Also, carvedilol increased cardiac levels of PIP2 and AKT activity and reduced 
DAG and caspase-3 activity compared to the $5 / 6$ nephrectomy group. These observations are in harmony with reduced cardiac remodeling and increased $\beta$-arrestin2 levels as illustrated before. In agreement with our findings, Zheng and colleagues showed that carvedilol reduced cardiac apoptosis in a rat model of diabetic cardiomyopathy as evidenced by increased AKT activity and reduced caspase-3 activity [36].

To explore the effect of GRK5 in conveying the cardioprotective role of carvedilol in 5/6 nephrectomized rats, we examined the effect of amlexanox (GRK5i) alone and in combination with carvedilol on all measured parameters.

GRK5 is a serine/threonine kinase that mediates GPCR phosphorylation after ligand binding and promotes membrane translocation of $\beta$-arrestins, receptor endocytosis, and $\beta$-arrestin signaling [12]. In the same context, GRK5 can directly activate the nuclear factor $\kappa \mathrm{B}(\mathrm{NF} \kappa \mathrm{B})$ protein [37]. Activation of $\beta$-arrestin signaling by GRK5 mediates anti-inflammatory effects [38], in contrast to $\mathrm{NF} \kappa \mathrm{B}$ which mediates inflammatory effects [39]. Therefore, GRK5 may promote opposing signals and the net result depends on the basal level of inflammation in the studied subjects [40].

In the current study, amlexanox use significantly ameliorated the detrimental changes in 5/6 nephrectomized rats. However, the effects observed were significantly lower than that of carvedilol. We think that the improvement observed with amlexanox may be due to its anti-inflammatory effect not due to inhibition of GRK5/ $\beta$-arrestin2 pathway.

Moreover, pre-injection of amlexanox significantly reduced the ameliorative effects of carvedilol in $5 / 6$ nephrectomized rats. This observation can be explained by the antagonism between amlexanox and carvedilol on the GRK5/ $\beta$ arrestin 2 level and support the potential role of the GRK5/ $\beta$-arrestin 2 pathway in this study.

\section{CONCLUSIONS}

Considering all previous findings, we showed that the cardioprotective effects of carvedilol in the cardiorenal syndrome are highly correlated with the upregulation of GRK5/ $\beta$-arrestin2 pathway.

\section{Funding}

This research did not receive any specific grant from funding agencies in the public, commercial, or not-for-profit sectors.

\section{Conflict of Interest Statement}

The authors have no conflicts of interest to declare.

\section{Author contributions}

all authors have participated in the concept and design, analysis and interpretation of data, drafting or revising of the manuscript, and that they have approved the manuscript as submitted.

\section{REFERENCES}

1. Rangaswami J, Bhalla V, Blair JE, Chang TI, Costa $\mathrm{S}$, Lentine $\mathrm{KL}$ et al. Cardiorenal syndrome: classification, pathophysiology, diagnosis, and treatment strategies: a scientific statement from the American Heart Association. Circulation. 2019 Apr 16;139(16): e840-78.

2. Suresh H, Arun BS, Moger V, Swamy M. Cardiorenal syndrome type 4: A study of cardiovascular diseases in chronic kidney disease. Indian Heart J. 2017; 69: 11-6.

3. Khoury S, Steinvil A, Gal-Oz A, Margolis G, Hochstatd A, Topilsky Y et al. Association between central venous pressure as assessed by echocardiography, left ventricular function and acute cardio-renal syndrome in patients with ST segment elevation myocardial infarction. Clin Res Cardiol. 2018; 107: 937-44.

4. Vinod P, Krishnappa V, Chauvin AM, Khare A, Raina R. Cardiorenal syndrome: role of arginine vasopressin and vaptans in heart failure. Cardiol Res. 2017; 8: 87

5. Carlstrom M, Montenegro MF. Therapeutic value of stimulating the nitrate-nitrite-nitric oxide pathway to attenuate oxidative stress and restore nitric oxide bioavailability in cardiorenal disease. J Intern Med. 2019; 285: 2-18.

6. Yu L, Huang B, Wang Z, Wang S, Wang M, Li X, et al. Impacts of renal sympathetic activation on atrial fibrillation: the potential role of the autonomic cross talk between kidney and heart. J Am Heart Assoc. 2017; 6: e004716.

7. Rajagopal S, Rajagopal K, Lefkowitz RJ. Teaching old receptors new tricks: biasing seventransmembrane receptors. Nat Rev Drug Discov. 2010; 9: 373

8. Soma K, Yao A, Saito A, Inaba T, Ishikawa Y, Hirata Y, et al. Regular Treatment Strategy with a Large Amount of Carvedilol for Heart Failure Improves Biventricular Systolic Failure in a Patient with Repaired Tetralogy of Fallot. Int Heart J. 2018; 17525.

9. Wisler JW, DeWire SM, Whalen EJ, Violin JD, 
Drake MT, Ahn S, et al. A unique mechanism of $\beta$ blocker action: carvedilol stimulates $\beta$-arrestin signaling. Proc Natl Acad Sci. 2007; 104: 16657-62.

10. Gutkind JS, Kostenis E. Arrestins as rheostats of GPCR signalling. Nat Rev Mol Cell Biol. 2018; 19 615.

11. Teixeira LB, Parreiras-e-Silva LT, BruderNascimento T, Duarte DA, Simões SC, Costa RM, et al. Ang-(1-7) is an endogenous $\beta$-arrestin-biased agonist of the AT 1 receptor with protective action in cardiac hypertrophy. Sci Rep. 2017; 7: 11903.

12. Shenoy SK, Drake MT, Nelson CD, Houtz DA, Xiao $\mathrm{K}$, Madabushi $\mathrm{S}$, et al. $\beta$-Arrestin-dependent, $\mathrm{G}$ protein-independent ERK1/2 activation by the $\beta 2$ adrenergic receptor. J Biol Chem. 2006; 281: 126173.

13. Toker A. The synthesis and cellular roles of phosphatidylinositol 4, 5-bisphosphate. Curr Opin Cell Biol. 1998; 10: 254-61.

14. Ibrahim IA, Nakaya M, Kurose H. Ezrin, Radixin, and Moesin Phosphorylation in NIH3T3 Cells Revealed Angiotensin II Type 1 Receptor CellType-Dependent Biased Signaling. J Pharmacol Sci. 2013; 122: 1-9.

15. Alessi DR, Andjelkovic M, Caudwell B, Cron P, Morrice N, Cohen P, et al. Mechanism of activation of protein kinase B by insulin and IGF-1. EMBO J. 1996; 15: 6541-51.

16. Mohamed RM, Morimoto S, Ibrahim IA, Zhan DY, Du CK, Arioka M, et al. GSK-3 $\beta$ heterozygous knockout is cardioprotective in a knockin mouse model of familial dilated cardiomyopathy. Am J Physiol Heart Circ Physiol. 2016; 310: H1808-15.

17. Rodriguez-Perez JC, Losada A, Anabitarte A, Cabrera J, Llobet J, Palop L, et al. Effects of the novel multiple-action agent carvedilol on severe nephrosclerosis in renal ablated rats. J Pharmacol Exp Ther. 1997; 283: 336-44.

18. Reilly SM, Chiang SH, Decker SJ, Chang L, Uhm M, Larsen MJ, et al. An inhibitor of the protein kinases TBK1 and IKK- $\varepsilon$ improves obesity-related metabolic dysfunctions in mice. Nat Med. 2013; 19 : 313.

19. Švíglerová J, Kuncova J, Nalos L, Tonar Z, Rajdl D, Stengl M. Cardiovascular parameters in rat model of chronic renal failure induced by subtotal nephrectomy. Physiol Res. 2010; 59.

20. Morrison LK, Harrison A, Krishnaswamy P, Kazanegra R, Clopton P, Maisel A. Utility of a rapid B-natriuretic peptide assay in differentiating congestive heart failure from lung disease in patients presenting with dyspnea. J Am Coll Cardiol. 2002; 39: 202-9.

21. Zoccali C, Mallamaci F, Maas R, Benedetto FA, Tripepi G, Malatino LS, et al. Left ventricular hypertrophy, cardiac remodeling and asymmetric dimethylarginine (ADMA) in hemodialysis patients. Kidney Int. 2002; 62: 339-45.

22. Ibrahim IA, Kurose H. $\beta$-arrestin-mediated signaling improves the efficacy of therapeutics. J Pharmacol Sci. 2012; 118: 408-12.

23. Meier KE, Sperling DM, Insel PA. Agonist-mediated regulation of alpha 1 -and beta 2 -adrenergic receptors in cloned MDCK cells. Am J Physiol Cell Physiol. 1985; 249: C69-77.

24. Ungerer M, Böhm M, Elce JS, Erdmann E, Lohse MJ. Altered expression of beta-adrenergic receptor kinase and beta 1-adrenergic receptors in the failing human heart. Circulation. 1993; 87: 454-63.

25. Ungerer M, Parruti G, Böhm M, Puzicha M, DeBlasi A, Erdmann E, et al. Expression of beta-arrestins and beta-adrenergic receptor kinases in the failing human heart. Circ Res. 1994;74: 206-13.

26. Vinge LE, Øie E, Andersson Y, Grøgaard HK, Andersen GØ, Attramadal H. Myocardial distribution and regulation of GRK and $\beta$-arrestin isoforms in congestive heart failure in rats. Am J Physiol Heart Circ Physiol. 2001; 281: H2490-9.

27. Philip JL, Razzaque MA, Han M, Li J, Theccanat T, $\mathrm{Xu} \mathrm{X}$, Akhter SA. Regulation of mitochondrial oxidative stress by $\beta$-arrestins in cultured human cardiac fibroblasts. Dis Model Mech. 2015; 8: 157989.

28. Park DO, Jhon DY, Lee CW, Lee KH, Rhee SG. Activation of phospholipase $\mathrm{C}$ isozymes by $\mathrm{G}$ protein beta gamma subunits. J Biol Chem. 1993; 268: 4573-6.

29. Newton AC. Regulation of protein kinase C. Curr Opin Cell Biol. 1997; 9: 161-7.

30. Takeishi Y, Ping P, Bolli R, Kirkpatrick DL, Hoit BD, Walsh RA. Transgenic overexpression of constitutively active protein kinase $\mathrm{C} \varepsilon$ causes concentric cardiac hypertrophy. Circ Res. 2000; 86: 1218-23.

31. Braz JC, Gregory K, Pathak A, Zhao W, Sahin B, Klevitsky $\mathrm{R}$, et al. PKC- $\alpha$ regulates cardiac contractility and propensity toward heart failure. Nat Med. 2004; 10: 248.

32. Osaki M, Oshimura MA, Ito H. PI3K-Akt pathway: its functions and alterations in human cancer. Apoptosis. 2004; 9: 667-76.

33. Chang H, Li C, Wang Q, Lu L, Zhang Q, Zhang Y, et al. QSKL protects against myocardial apoptosis on heart failure via PI3K/Akt-p53 signaling pathway. Sci Rep. 2017; 7: 16986.

34. Ajam T, Ajam S, Devaraj S, Fudim M, Kamalesh M. Effect on Mortality of Higher Versus Lower $\beta$ Blocker (Metoprolol Succinate or Carvedilol) Dose in Patients With Heart Failure. Am J Cardiol. 2018; 122: 994-8.

35. Aarons RD, Nies AS, Gal JO, Hegstrand LR, Molinoff PB. Elevation of beta-adrenergic receptor density in human lymphocytes after propranolol administration. J Clin Invest. 1980; 65: 949-57.

36. Zheng W, Shang X, Zhang C, Gao X, Robinson B, Liu J. The Effects of Carvedilol on Cardiac Function and the AKT/XIAP Signaling Pathway in Diabetic Cardiomyopathy Rats. Cardiology. 2017; 136: 20411.

37. Zhang Y, Shen GL, Shangguan LJ, Yu Y, He ML. Involvement of $\mathrm{NF} \kappa \mathrm{B}$ signaling in mediating the effects of GRK5 on neural stem cells. Brain Res. 2015; 1608: 31-9.

38. Du RW, Du RH, Bu WG. $\beta$-Arrestin 2 mediates the 
anti-inflammatory effects of fluoxetine in lipopolysaccharide-stimulated microglial cells. J Neuroimmune Pharmacol. 2014; 9: 582-90.

39. Barroso M, Kao D, Blom HJ, de Almeida IT, Castro $\mathrm{R}$, Loscalzo J, et al. S-adenosylhomocysteine induces inflammation through NFkB: A possible role for $\mathrm{EZH} 2$ in endothelial cell activation. Biochim Biophys Acta Molr Basis Dis. 2016; 1862:82-92.

40. Oral EA, Reilly SM, Gomez AV, Meral R, Butz L, Ajluni $\mathrm{N}$, et al. Inhibition of $\mathrm{IKK} \varepsilon$ and $\mathrm{TBK} 1$ improves glucose control in a subset of patients with type 2 diabetes. Cell Metabol. 2017; 26: 157-70.

\section{To Cite}

Mohamed, R., Elshazly, S., Salem, A., Mahmoud, N. Role of GRK5/ $\beta$-arrestin2 pathway in mediating the cardioprotective effects of carvedilol in 5/6 nephrectomized rats. Zagazig University Medical Journal, 2022; (163-173): -. doi: 10.21608/zumj.2021.77150.2238 